\title{
A Case of Ruptured Rudimentary Horn of Uterus during Pregnancy
}

\author{
RUMAN ${ }^{1}$, KHANAM K ${ }^{2}$ AKHTER $S^{3}$, KARIM R $^{4}$
}

\begin{abstract}
:
Pregnancy in a rudimentary horn of an unicornuate uterus is a rare and life threatening situation for mother and fetus. Usually pregnancy continues upto approximately 18-20 weeks of gestational age. Then it usually ruptures and severe haemorrhage ensues. Emergency laparotomy is the treatment of choice. Here we report a case of 36 years woman with secondary subfertility who has history of taking ovulation inducing drug. She presented with 20 weeks amenorrhoea with severe abdominal pain and hypovolemic shock. Urgent laparotomy was done and the diagnosis was confirmed.
\end{abstract}

\section{Introduction:}

Rudimentary horn pregnancy (RHP) is a very rare and grave condition which has an incidence approximately 1 per 100,000 to 140,000 pregnancies ${ }^{1}$. Here we present a case of ruptured RHP with hypovolemic shock in a woman with secondary subfertility.

\section{Case summary:}

A 28 years old lady $2^{\text {nd }}$ gravida, para $1+0$, got herself in emergency casualty department of Dhaka Combined Military Hospital (CMH) with complains of 4 months pregnancy on $14^{\text {th }}$ April, 2016. She was having severe abdominal pain for one day .It was also associated with sudden onset of difficulty in micturition and constipation for last two days. At presentation, she was pale, pulse $130 \mathrm{bpm}$, BP was $80 / 50 \mathrm{~mm}$ of $\mathrm{Hg}$. Abdomen was hugely distended and there was generalized tenderness in abdomen. No vaginal bleeding was there.

She was mother of one child who is 11 years old. As she was suffering from secondary subfertility, she took ovulation inducing drug for two month and became amenorrhic 4 months back. Urine strip test was positive for pregnancy but since then she has never took a single antenatal check up.

Her hemoglobin level was $5.0 \mathrm{gm} / \mathrm{dl}$. Ultrasonogram (USG) of whole abdomen showed huge collection in peritoneal cavity and uterine cavity was empty [Fig:1] with a small 20 weeks size dead fetus floating there outside uterus [Fig: 2]. Urgent decision for emergency laparotomy was taken with blood keeping ready.

Abdomen was opened by right paramedian incision. Huge amount of blood (around two litres) was there in the peritoneal cavity which was sucked out. A small dead fetus was found in the pouch of Douglas. There was a left rudimentary horn of the uterus with rupture on the superior margin. The placenta was still within the uterine horn. The left fallopian tube was of normal length and attached to the rudimentary horn[Fig. 2]. Left ovary was normal and attached by its ligament to the rudimentary horn. The uterus was of normal size with the right fallopian tube and right ovary. Ovaries on both side was healthy.

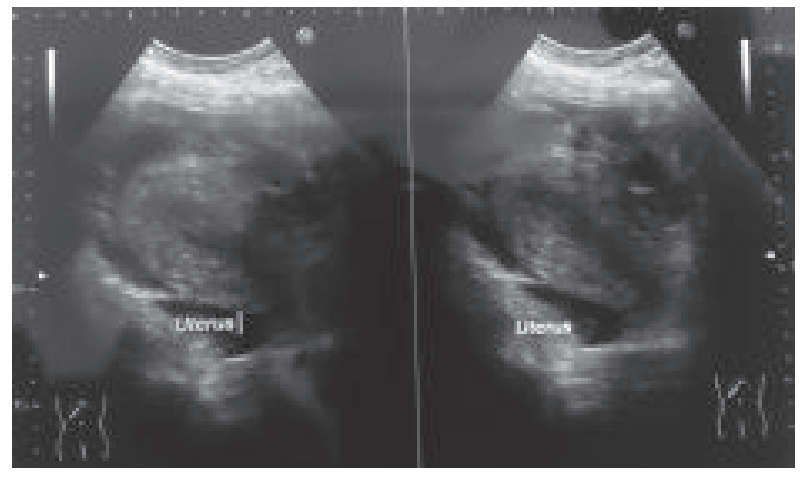

Fig.-1: USG showing empty uterine cavity.

1. Major, Classified Specialist, Obstetrics and Gynaecology, $\mathrm{CMH}$, Dhaka, Bangladesh.

2. Colonel, Advisor Specialist, Obstetrics and Gynaecology, $\mathrm{CMH}$, Dhaka, Bangladesh.

3. Lt.Colonel, Classified Specialist, Obstetrics and Gynaecology, $\mathrm{CMH}$, Dhaka, Bangladesh.

4. Major, Classified Specialist, Obstetrics and Gynaecology, CMH, Dhaka, Bangladesh.

Address of Correspondence: Ruman U, Major, Classified Specialist, Obstetrics and Gynaecology, CMH, Dhaka, Bangladesh. 


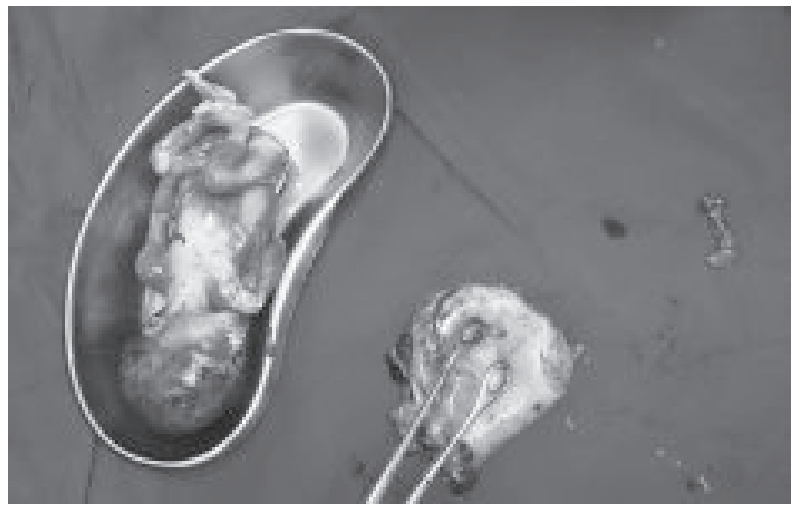

Fig.-2: Excised rudimentary horn with dead fetus.

Rudimentary horn was excised and uterus was repaired. Left salpingectomy was done. Patient was transfused with two units of blood during operation. Her postoperative period was uneventful. She was discharged on $10^{\text {th }}$ postoperative day.

\section{Discussion:}

According to American Society for Reproductive Medicine Classification of mullerian Anomalies (1988), Non communicating rudimentary horn of an unicornuate uterus falls in class II (unicornuate) anomaly ${ }^{2}$. Here, mullerian duct of one side fails to develop completely on one side and fail to communicate with other side as well. Presence of non communicating horn is commoner than other forms of unicornuate uterus and it estimates almost about in $83 \%{ }^{3}$.

In a non communicating horn pregnancy is usually possible by transperitoneal migration of sperm or fertilized ovum ${ }^{4}$. Almost $90 \%$ of pregnancy ends up in rupture in and around 20 weeks. Timing of rupture depends on ability of surrounding uterine wall to support and supply to the fetus. As the wall of uterus is thick, muscular and rich in vascular supply, when it ruptures, causes massive haemorrhage and haemoperitonium ${ }^{5}$. Though there is huge blood loss, maternal mortality is low which is opposite to increased fetal demise in these cases due to failure to reach the viable age in most cases ${ }^{6}$.

It is not common to make a pre rupture diagnosis of a rudimentary horn pregnancy. It is often missed in routine ultrasound scan and difficulties increased with advanced gestational age ${ }^{7}$. It can be misdiagnosed as tubal, abdominal, cornual and intrauterine pregnancy. Though USG has a sensitivity of $26 \%$, it is held as a useful diagnostic tool. Others are MRI, hysterosalpingography, laparoscopy ${ }^{8}$.
Though many cases are misdiagnosed or lately diagnosed, Tsafrir et al. reported 2 cases of rudimentary horn pregnancy found in the first trimester by sonography and confirmed by MRI. They outlined a set of criteria for diagnosing pregnancy in the rudimentary horn. They are (1) a pseudo pattern of asymmetrical bicornuate uterus; (2) absent visual continuity tissue surrounding the gestation sac and the uterine cervix; (3) presence of myometrial tissue surrounding the gestational sac ${ }^{9}$. Cases with late diagnosis leads to rupture of horn and catastrophic haemorrhage as we have seen in our case.

Immediate surgical excision of ruptured horn is usually recommended even if it is diagnosed before rupture to avoid emergency situation ${ }^{7}$. Removal can be through laparoscopy or laparotomy. Early diagnosis can be privileged by use of methotrexate and removal of rudimentary horn ${ }^{10}$. Pregnancy progressing to third trimester is reported in one case ${ }^{7}$. Repeated failed attempt of induction should arise a suspicion of extra uterine pregnancy. A case showed three failed surgical termination in a single pregnancy ended up with rupture of rudimentary horn ${ }^{11}$.

\section{Conclusion:}

Rudimentary horn rupture is a grave situation for both mother and fetus. High degree of suspicion is required for the diagnosis. Early detection prior to rupture is life-saving.

\section{References:}

1. Hassan C. H. C, Kadir A, Karim A, Ismail N. A. $\mathrm{M}$, and Omar M. H, "Case report of ruptured non-communicating right rudimentary horn pregnancy: an acute emergency," Acta Medica, 2011;54( 3):125-26.

2. Pratap K, Malhotra N . Jeffcoat's Principles of Gynaecology.7TH ed. New Delhi: Jaypee Brothers Medical Publishers(P) Itd;2008.

3. Heinonen PK. Unicornuate uterus and rudimentary horn. Fertil steril,1997; 68: 224-30.

4. Panayotidis $C$, Abdel-Fattah M, Leggott $M$ : Rupture of rudimentary horn of a unicornuate uterus at 15 weeks gestation. J Obstet Gynaecol. 2004, 24: 323-24.

5. Chowdhury S, Chowdhury T, and Azim E,. "Pregnancy in a non-communicating rudimentary horn of uterus: a clinical case 
report," Bangladesh Medical Journal. 2010; 39(1) :47-48.

6. Nahum G, "Rudimentary uterine horn pregnancy: the 20th-century worldwide experience of 588 cases," Journal of Reproductive Medicine for the Obstetrician and Gynecologist. 2002; 47( 2):151-63.

7. Jayasinghe $Y$, Rane A, Stalewski $H$, Grover S."The presentation and early diagnosis of the rudimentary uterine horn." Obstetrics and Gynecology. 2005; 105(61):456-67.

8. Lawhon B. P, Wax J. R, and Dufort R. T, "Rudimentary uterine horn pregnancy diagnosed with magnetic resonance imaging" .Obstetrics and Gynecology.1998;91( 5):869.
9. Tsafrir A, Rojansky N, Sela H. Y, Gomori J. M, and Nadjari M, "Rudimentary horn pregnancy: first-trimester prerupture sonographic diagnosis and confirmation by magnetic resonance imaging," Journal of Ultrasound in Medicine. 2005; 24(2):219-23.

10. Edelman A. B, Jensen J. T, Lee D. M, and Nichols M. D, "Successful medical abortion of a pregnancy within a noncommunicating rudimentary uterine horn." American Journal of Obstetrics and Gynecology. 2003; 189(3) 88687.

11. Okonta P, Abedi H, Ajuyah C, Omo-Aghoja L.Pregnancy in a noncommunicating rudimentary horn of a unicornuate uterus: a case report . Cases Journal.2009;2: 6624. 